\title{
Hippocampal HDAC4 Contributes to Postnatal Fluoxetine-Evoked Depression-Like Behavior
}

\author{
Ambalika Sarkar', Parul Chachra',3, Pamela Kennedy ${ }^{2,3}$, Catherine J Pena², Lynette A Desouza', \\ Eric J Nestler ${ }^{2}$ and Vidita A Vaidya*, I \\ 'Department of Biological Sciences, Tata Institute of Fundamental Research, Mumbai, India; ${ }^{2}$ Fishberg Department of Neuroscience, Friedman \\ Brain Institute, Icahn School of Medicine at Mount Sinai, New York, NY, USA
}

\begin{abstract}
Fluoxetine treatment in adulthood evokes antidepressant and anxiolytic responses. Paradoxically, postnatal fluoxetine (PNFlx) induces persistent depression- and anxiety-like behaviors. The mechanistic underpinnings of this paradox remain poorly understood. Here, we examined specific molecular changes in the rat hippocampus that accompany perturbed emotionality observed across life following PNFIx. PNFIx-induced hippocampal gene regulation observed in microarray and quantitative PCR studies indicate functional enrichment of genes involved in response to organic substances, protein kinase pathways, DNA binding, and transcriptional repression. We noted specific transcripts (Hdac4, mammalian target of rapamycin (mTOR), Gnail, protein kinase C gamma (Prkcc), and hyperpolarizationactivated cyclic nucleotide-gated channel I $(\mathrm{Hcn} /)$ ) that were consistently dysregulated across life, and selectively influenced by postnatal, but not adult, fluoxetine. Increased histone deacetylase-4 (HDAC4) recruitment, accompanied by decreased activating histone acetylation marks at the mTOR and Gnail promoters, indicate a role for HDAC4 in PNFlx-mediated gene dysregulation. Strikingly, coadministration of the HDAC inhibitor sodium butyrate with PNFlx prevented the dysregulation of Hdac4 and mTOR, and the emergence of depression- and anxiety-like behavior. Importantly, we also find that retreatment of PNFlx animals with fluoxetine in adulthood reversed the increased Hdac4 expression, prevented HDAC4 recruitment to the mTOR and Gnail promoters, and attenuated the decline in mTOR and Gnail expression, coincident with normalization of PNFlx-evoked depression- and anxiety-like behavior. Further, we show that viral-mediated hippocampal overexpression of Hdac4 was sufficient to induce depression-, but not anxiety-, like behavior in adulthood. Our results highlight the unique nature of molecular signatures evoked by PNFlx, and implicate HDAC4 in the dysregulated gene expression and emergence of perturbed emotionality following fluoxetine exposure in early life.

Neuropsychopharmacology (20I4) 39, 222 I-2232; doi:I0.1038/npp.2014.73; published online 23 April 20I4
\end{abstract}

\section{INTRODUCTION}

Fluoxetine (Prozac), a selective serotonin reuptake inhibitor (SSRI), is the drug of choice for gestational/postpartum depression (Ward and Zamorski, 2002) and children/ adolescents with mood disorders (Kodish et al, 2011), given its perceived favorable risk to benefit ratio. However, preclinical and clinical studies have raised concerns about early fluoxetine exposure (Ansorge et al, 2004; Olivier et al, 2011). Studies in mouse models indicate enhanced depression- and anxiety-like behavior following postnatal SSRI administration (Ansorge et al, 2004, 2008; Karpova et al, 2009; Popa et al, 2008). Perinatal SSRI administration disrupts sleep (Popa et al, 2008), circadian rhythms (Kiryanova et al, 2013), stress responses (Norcross et al, 2008), and serotonin neurocircuitry (Silva et al, 2010;

*Correspondence: Dr VA Vaidya, Department of Biological Sciences, Tata Institute of Fundamental Research, Homi Bhabha Road, Mumbai 400005, India, Tel: +9| 22 22782608, Fax: +91 22 228046।0,

E-mail:vvaidya@tifr.res.in

${ }^{3}$ These authors contributed equally to this work.

Received 16 October 2013; revised 28 February 2014; accepted 19

March 2014; accepted article preview online 25 March 2014
Maciag et al, 2006). Similarly, deletion of the serotonin transporter, the molecular target of SSRIs, early in life induces depression- and anxiety-like symptoms in mice (Holmes et al, 2003; Lira et al, 2003). Clinical evidence supports an association between pediatric and adolescent fluoxetine usage and adverse effects on emotionality (Olivier et al, 2011). In contrast, adult fluoxetine (AFlx) induces antidepressant and anxiolytic responses in patients (Brambilla et al, 2005; Olivier et al, 2011) and animal models (Dulawa et al, 2004). These studies suggest opposing effects of fluoxetine on emotionality based on the temporal window of administration, for which the underlying mechanisms are unknown.

We hypothesized that depression- and anxiety-like behaviors in animals with a history of postnatal fluoxetine (PNFlx) involve underlying molecular changes maintained long after drug treatment. We examined PNFlx-induced molecular changes in the hippocampus, a limbic structure implicated in the regulation of mood (Deacon et al, 2002; Kheirbek et al, 2013) and known to transiently express the serotonin transporter during postnatal life (Narboux-Nême et al, 2008). We identified unique molecular signatures associated with postnatal, but not adult, fluoxetine treat- 
ment, including a persistent upregulation of the histonemodifying enzyme, Hdac4 (histone deacetylase-4). PNFlxevoked depression- and anxiety-like behavior, and specific molecular signatures were prevented by HDAC inhibitor cotreatment during postnatal life, and also normalized by fluoxetine retreatment in adulthood. Further, hippocampal HDAC4 overexpression was sufficient to induce depressionlike behavior. Our findings implicate hippocampal HDAC4 as a mediator of the persistent increases in depression-like behavior following PNFlx.

\section{MATERIALS AND METHODS}

\begin{abstract}
Animals
Male Sprague-Dawley rats bred at the Tata Institute of Fundamental Research (TIFR) animal facility were used for experiments and maintained with ad libitum access to food and water. Procedures followed the National Institutes of Health Guide for the Care and Use of Animals, and were approved by the TIFR Animal Ethics Committee. Viral overexpression experiments were performed at the Mount Sinai School of Medicine and were approved by the Institutional Animal Care and Use Committee.
\end{abstract}

\section{Drug Treatments}

PNFlx treatment involved oral fluoxetine $(10 \mathrm{mg} / \mathrm{kg}$, Sigma, USA) or vehicle ( $5 \%$ sucrose) administration to rat pups from postnatal day (P) 2 to P21. AFlx treatment involved once daily intraperitoneal administration of fluoxetine $(10 \mathrm{mg} / \mathrm{kg})$ or vehicle (saline) for 21 days to 2 -month-old animals. To address whether AFlx reversed PNFlx-evoked changes there were three groups: control (Ctrl), PNFlx, and PNFlx + AFlx. Behavioral experiments commenced after 21 days of AFlx and fluoxetine treatment was continued through behavioral experiments. For HDAC inhibitor sodium butyrate $(\mathrm{SB})$ experiments, pups received fluoxetine $(10 \mathrm{mg} / \mathrm{kg})$, fluoxetine $(10 \mathrm{mg} / \mathrm{kg})+\mathrm{SB}(300 \mathrm{mg} / \mathrm{kg}$, Sigma $)$, or vehicle (5\% sucrose) orally from P2 to 21 with three groups: Ctrl, PNFlx, and PNFlx + PNSB. Drug doses were based on prior literature (Dulawa et al, 2004; Ansorge et al, 2008; Kim et al, 2009).

\section{Behavioral Tests}

Behavioral tests were performed at specific time points across life. Pair-wise social play was assessed in Ctrl and PNFlx rats (P28-P32; $n=8-10$ per group) by an investigator blind to treatment conditions (Homberg et al, 2007). Depression- and anxiety-like behavior in Ctrl and PNFlx groups ( 2 months; $n=8-10$ per group) were assessed using the modified forced swim test (FST), open field test (OFT), and elevated plus maze (EPM). Further, Ctrl and PNFlx animals at 18 months of age ( $n=8-10$ per group), postnatal SB experiment ( $n=6-12$ per group), AFlx retreatment experiment ( $n=6-10$ per group), and hippocampal HDAC4 overexpression experiment ( $n=6-7$ per group) were tested on the OFT and FST. For detailed methods and behavioral paradigms (Supplementary Figure S1), see Supplementary Information. Behaviors were analyzed using Ethovision 3.1 (Noldus, the Netherlands).

\section{Microarray Experiments}

Microarray analysis with a custom rat array $8 \times 15 \mathrm{~K}$ was performed on hippocampi from control and PNFlx animals (2 months, $n=4$ per group) as previously described (Benekareddy et al, 2010). Data were extracted using Agilent Feature Extraction software v 10.5.1.1 and differentially regulated genes were filtered ( $>1.35$ for upregulation and $<0.74$ for downregulation). Statistical analysis was performed using $t$-test $(p<0.05)$ with multiple comparison corrections (Benjamini and Hochberg, 1995). Array data are deposited in the NCBI Gene Expression Omnibus (Accession number GSE42940). Functional analysis was performed using DAVID (Database for Annotation Visualization and Integrated Discovery) on array data after removal of odorant receptors as potential false positives to avoid any bias to gene ontology analysis (Dennis et al, 2003).

\section{Chromatin Immunoprecipitation Assay}

ChIP analysis was performed as previously described (Renthal et al, 2007). ChIP assays measured H3 (H3ac) and $\mathrm{H} 4(\mathrm{H} 4 \mathrm{ac})$ acetylation, and HDAC4 enrichment at gene promoters. Chromatin enriched for $\mathrm{H} 3 \mathrm{ac}, \mathrm{H} 4 \mathrm{ac}$, and HDAC4 was subjected to quantitative PCR (qPCR) analysis (see Supplementary Information).

\section{Quantitative PCR}

qPCR was performed on Ctrl and PNFlx (P21, 2 months and 18 months; $n=8-10$ per group); Ctrl and AFlx $(n=6-10$ per group); Ctrl, PNFlx, and PNFlx + AFlx $(n=6-11$ per group); Ctrl, PNFlx, and PNFlx + PNSB $(n=6-10$ per group), HSV-GFP and HSV-HDAC4-GFP ( $n=4$ per group); and qPCR for ChIP analysis in Ctrl, PNFlx, and PNFlx + AFlx ( $n=6-10$ per group). Reverse transcribed RNA was subjected to qPCR using primers for genes of interest (Supplementary Figures S2 and S3). Quantification was done using the $\Delta \Delta \mathrm{Ct}$ method as previously described (Bookout and Mangelsdorf, 2003) and data were normalized to hypoxanthine-guanine phosphoribosyltransferase (Hprt1), which was not regulated by any of the treatments used in this study. Results were compared with the controls and expressed as fold change \pm SEM.

\section{Western Blot Analysis}

Hippocampi were lysed and separated by SDS-PAGE, and transferred to polyvinyldifluoride membranes (GE Healthcare, Buckinghamshire, UK). Membranes were blocked, incubated with rabbit anti-mTOR (1:1000, Cell Signaling Technologies), rabbit anti-HDAC4 (1:500, Abcam, UK), rabbit anti-GAPDH (1:1000, Cell Signaling Technologies), and rabbit anti- $\beta$ III-tubulin antibody (1:1000; Covance, Princeton, NJ) before incubation with a horseradish peroxidase-conjugated goat anti-rabbit secondary antibody (GE Healthcare) and detection with an ECL substrate (GE Healthcare). Densitometric measurements were performed using a Macintosh-based Scion Image software (Scion, USA) and levels of mTOR and HDAC4 were normalized to $\beta$ IIItubulin and GAPDH, respectively. 


\section{Viral-Mediated Gene Transfer}

A bicistronic HSV-HDAC4-GFP and HSV-GFP (Clark et al, 2002; Renthal et al, 2007) virus with expression driven off an IE4/5 promoter (HDAC4 cDNA was a kind gift of E Olson, University of Texas Southwestern Medical Center) were bilaterally delivered into the hippocampus of treatment-naive adult rats as previously described (see Supplementary Information). Viral targeting and overexpression were assessed by imaging GFP fluorescence, qPCR, and western blot analysis.

\section{Statistical Analysis}

Experiments with two or three groups were analyzed using unpaired Student's $t$-test or one-way ANOVA followed by Student-Newman-Keul's post hoc test, respectively, with significance at $p<0.05$ (Instat, Graphpad Software, USA).

\section{RESULTS}

\section{PNFlx Evokes Persistent Depression- and Anxiety-Like Behavior}

We examined the consequences of PNFlx treatment on juvenile play (P28-32) and depression- and anxiety-like behavior in adulthood and at 18 months of age (Figure 1a, Supplementary Figure S1). Adolescent PNFlx animals showed decreased social grooming and frequency of pouncing (Figure $1 \mathrm{~b}$ and $\mathrm{c}$ ), whereas follow and chase was unaltered (Figure 1d). PNFlx animals in adulthood showed depression- and anxiety-like behavior on the FST (Figure 1e-g), OFT (Figure $1 \mathrm{~h}-\mathrm{j}$ ), and EPM (Supplementary Figure S5). We noted increased immobility time, decreased latency to the first immobility event, and a trend $(p=0.07)$ toward increased immobility events on the FST in PNFlx animals. On the OFT, PNFlx animals exhibited an increased latency to approach the center, decreased percent time and path length in the center of the arena. Total distance traversed in the OFT was unaltered (Supplementary Figure S4). Depressionand anxiety-like behavior on the FST (Figure $1 \mathrm{k}$ and 1 , Supplementary Figure S6) and OFT (Figure 1m, Supplementary Figure S6) persisted up to 18 months in PNFlx animals. We observed no change in hippocampal-dependent spatial learning in PNFlx animals in adulthood (Supplementary Figure S7). Our results indicate that mood-related behavioral changes emerge as early as adolescence in PNFlx animals, and persist up to 18 months of age.

\section{PNFlx Alters the Hippocampal Transcriptome}

Microarray analysis on hippocampi from 2-month-old PNFlx animals (Figure 1n) revealed significant enrichment for genes associated with response to organic substances and glucocorticoid stimuli, regulation of cellular protein metabolic processes and protein kinase activity, DNA binding, and transcription repressor activity (Figure 1o, Supplementary Figure S8). We noted a preponderance of downregulated genes (771), as compared with those exhibiting a significant upregulation (519). qPCR validation in an independent cohort of 2-month-old PNFlx animals (Figure 2a) indicated strong reproducibility of our micro- array results (Figure 2c). qPCR analysis confirmed the regulation of multiple array-identified targets, including Hdac4, mTOR, Prkcc, Gnail, and Hcn1.

\section{PNFlx Induces Dysregulation of Specific Genes in the Hippocampus Across Life}

We next examined whether the PNFlx-evoked gene dysregulation in adulthood was observed across life, in keeping with the persistent alterations in behavior. Strikingly, Hdac4 expression was significantly upregulated at all three ages examined (P21, 2 months and 18 months) in PNFlx animals (Figure 2a-d). Although other upregulated genes validated in 2-month-old PNFlx animals did not exhibit a stable pattern, specific downregulated genes ( $m T O R$, Gnail, Prkcc, and Hcn1) showed a persistent decline in expression across life (Figure 2b-d). To address whether Hdac4, mTOR, Gnail, Prkcc, and Hcnl gene regulation was unique to PNFlx treatment, which evokes depression- and anxiety-like behavior, we examined the influence of AFlx known to induce antidepressant and anxiolytic responses on the expression of these genes. AFlx treatment did not alter hippocampal Hdac4, mTOR, Gnai1, Prkcc, or Hcn 1 expression (Figure 2e and f). Conversely, PNFlx did not influence hippocampal Bdnf, TrkB, Gdnf, $c$-Fos, or Arc expression, which are regulated following AFlx (Nibuya et al, 1995; Hisaoka et al, 2001; Miller et al, 2008; Figure 2g). The effects of PNFlx were selective to Hdac4 with no change observed in other Hdacs examined (Figure 2h). The enhanced Hdac4 expression in PNFlx animals in adulthood was accompanied by a significant enrichment of H3ac and $\mathrm{H} 4 \mathrm{ac}$ at the Hdac4 promoter (Figure 2i). These results implicate persistent dysregulation of Hdac4, mTOR, Gnail, Prkcc, and Hcn 1 as molecular signatures that accompany the life-long behavioral changes evoked by PNFlx.

As we observed a persistent decline in mTOR, Gnail, Prkcc, and Hcn 1 expression in PNFlx animals, we asked whether HDAC4, a potent transcriptional repressor, contributes to the decline in expression of these genes. ChIP analysis indicated significant HDAC4 enrichment at $m$ TOR and Gnail (Figure 2j), but not Prkcc and Hcn1 (Supplementary Figure S9), promoters following PNFlx. HDAC4 enrichment was accompanied by a significant decline in $\mathrm{H} 4 \mathrm{ac}$ at $m$ TOR and Gnail (Figure 2k), but not $\mathrm{H} 3 \mathrm{ac}$ at the $m T O R$ and Gnail promoters (Supplementary Figure S10).

\section{Postnatal Treatment with SB Prevents the Emergence of PNFlx-Evoked Molecular and Behavioral Changes}

To test the hypothesis that HDAC4 may contribute to PNFlx-evoked behavioral outcomes, animals were coadministered the HDAC inhibitor, SB, with fluoxetine in postnatal life. We profiled depression- and anxiety-like behavior and gene expression changes in adulthood (Figure 3a). Combined PNFlx and SB treatment (PNFlx + PNSB) prevented the emergence of depression- and anxiety-like behavior on the FST (Figure $3 \mathrm{~b}-\mathrm{e}$ ) and OFT (Figure $3 \mathrm{f}-\mathrm{j}$ ). One-way ANOVA and post hoc analysis indicated that the PNFlx + PNSB group differed significantly from PNFlx animals on immobility time (Figure 3c) and latency to first immobility (Figure 3d), with no differences on immobility events (Figure 3e). On the OFT, the PNFlx + PNSB group did not 

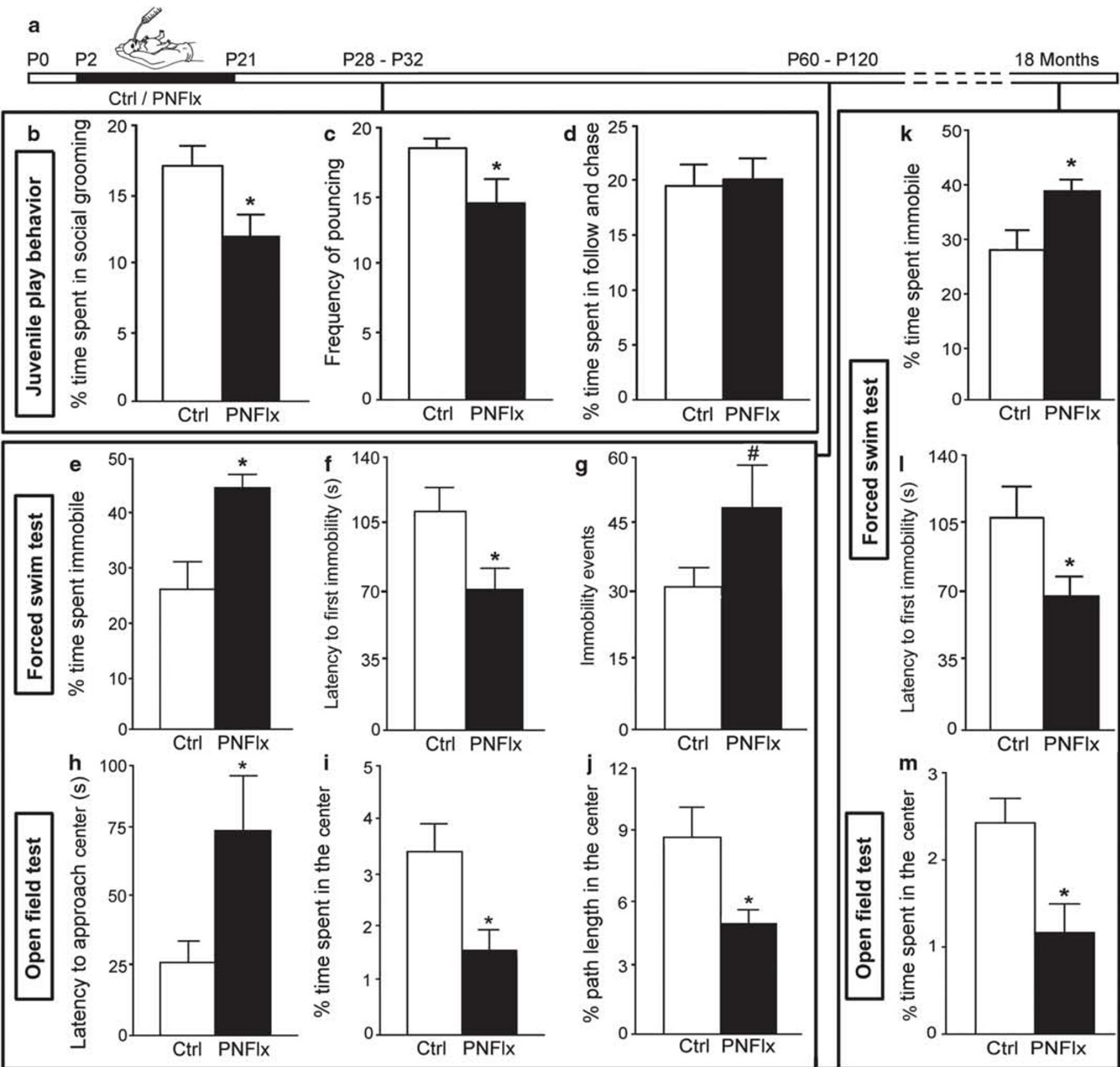

$$
\mathrm{P} 120
$$
ニニー-ン 18 Months
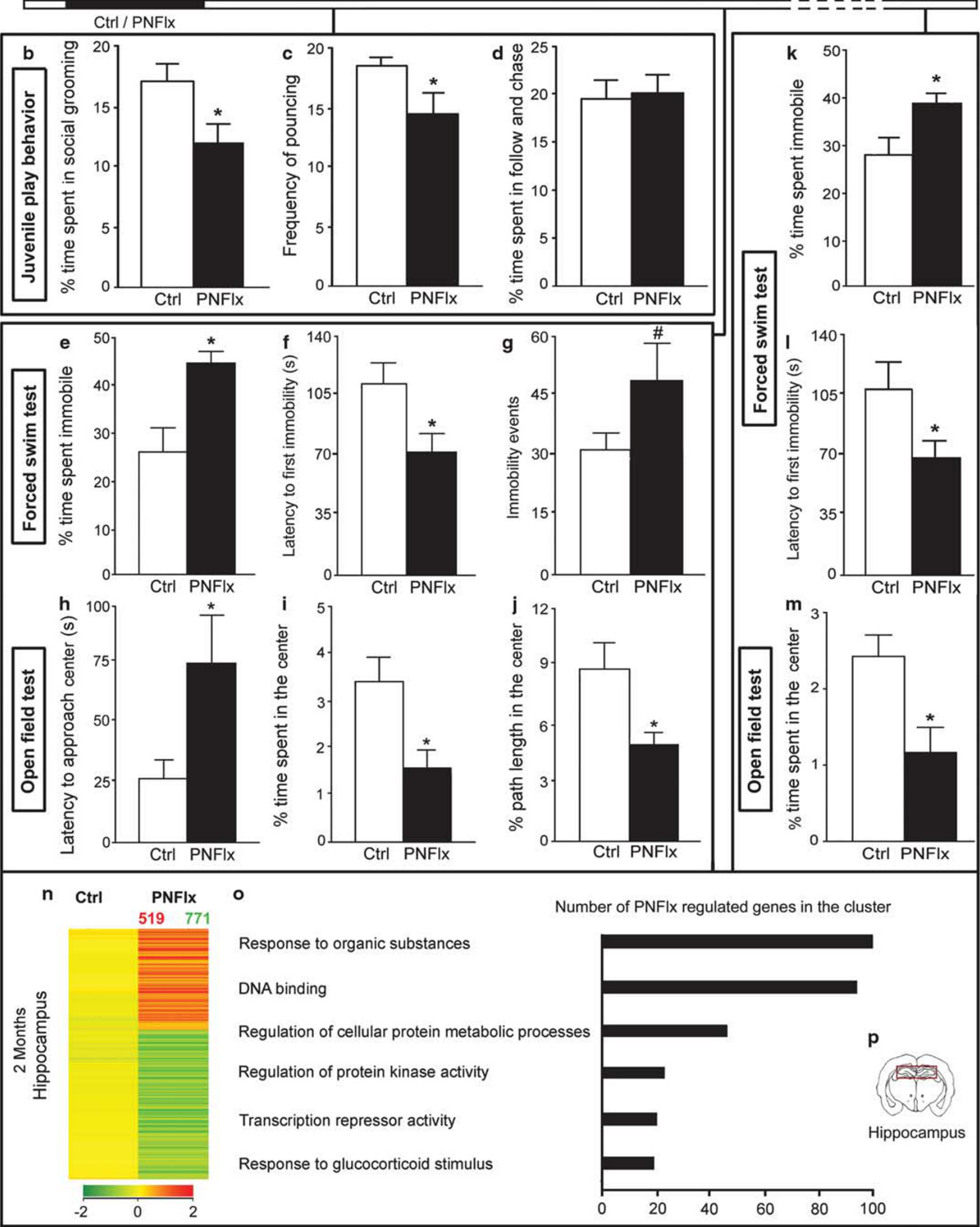
show decreases in percent time, path length, and number of visits to the center of the arena observed in PNFlx animals, and were similar to controls (Figure $3 \mathrm{f}-\mathrm{j}$ ). A history of PNSB treatment itself did not alter behavior on the OFT or FST tasks (Supplementary Figure S11) in adulthood.

We examined whether SB treatment could similarly prevent the PNFlx-mediated dysregulation of specific genes within the hippocampus. PNFlx + PNSB animals did not exhibit Hdac4 upregulation (Figure 3k) and failed to show the PNFlx-associated $m$ TOR decline (Figure 31). In contrast, decreased Gnai1, Prkcc, and Hcn1 expression (Figure 3m-o) was still observed in the PNFlx + PNSB group. These results indicate that concomitant HDAC inhibition prevents the emergence of PNFlx-evoked depression- and anxiety-like behaviors, as well as the regulation of specific genes.

\section{AFlx Treatment Reverses Behavioral and Molecular Signatures Associated with PNFlx}

Given that PNFlx and AFlx evoke distinct molecular and behavioral changes, we examined whether retreatment with fluoxetine in adulthood could influence PNFlx-evoked outcomes (Figure 4a). One-way ANOVA analysis indicated that AFlx completely reversed the PNFlx-evoked depression- and anxiety-like behaviors in the FST and OFT (Figure 4b-e). Further, PNFlx + AFlx animals exhibited antidepressant-like FST behaviors with decreased percent immobility and immobility events (Figure $4 \mathrm{~b}$ and c). Enhanced histone acetylation at the Hdac4 promoter and increased hippocampal Hdac4 mRNA were reversed to control levels in PNFlx + AFlx animals (Figure 4f and g). PNFlx + AFlx animals did not exhibit increased HDAC4 recruitment at the Gnail and $m$ TOR promoters (Figure $4 \mathrm{~h}$ and $\mathrm{j}$ ), and showed attenuation of the PNFlx-evoked decline in Gnail and mTOR (Figure 4i and k) expression. Western blot analysis revealed decreased mTOR levels in hippocampi from PNFlx animals, which was ameliorated in the PNFlx + AFlx group (Figure 4l). These results indicate that retreatment with fluoxetine in adulthood rescues behavioral and molecular changes evoked by PNFlx.

\section{Hippocampal Hdac4 Overexpression in Adulthood Evokes Depression-Like Behavior}

We next examined whether viral HDAC4 overexpression in adulthood could recapitulate PNFlx-evoked behavioral phenotypes. We performed bilateral stereotactic delivery of HSV-HDAC4-GFP or HSV-GFP into hippocampi of treatment-naive adult animals and assessed OFT and FST behavior (Figure 5a). HSV-HDAC4-GFP localization was confirmed by GFP fluorescence (Figure $5 b$ and c). HDAC4 overexpression was indicated by a strong trend toward significance in both qPCR $(p=0.07)$ and western blot $(p=0.062)$ analysis (Figure $5 \mathrm{~d}$ and e). Hippocampal HDAC4 overexpression evoked a significant decline in Gnail expression and a strong trend $(p=0.051)$ toward a decline in $m T O R$ expression (Figure $5 \mathrm{~d}$ ).

HSV-HDAC4-GFP animals exhibited enhanced percent immobility, decreased latency to first immobility, and a strong trend $(p=0.07)$ for increased immobility events (Figure 5f-h). On the OFT, the HSV-HDAC4-GFP group did not differ from controls (percent time: HSV-GFP $=5.27 \pm$ 0.86 , HSV-HDAC4-GFP $=9.49 \pm 2.49$; percent path length: $\mathrm{HSV}-\mathrm{GFP}=5.76 \pm 0.79, \quad$ HSV-HDAC4-GFP $=7.93 \pm 3.10$; number of visits: HSV-GFP $=28.8 \pm 4.7$, HSV-HDAC4$\mathrm{GFP}=34.2 \pm 12$; data are the mean \pm SEM, $p>0.05$, Student's $t$-test). These results indicate that enhanced hippocampal HDAC4 expression in adulthood is sufficient to evoke depression-, but not anxiety-, like behavior.

\section{DISCUSSION}

The major novel finding of our study is the identification of specific molecular signatures within the hippocampus that are associated with and contribute to the depression- and anxiety-like behaviors observed in PNFlx animals. PNFlx evoked persistent and unique gene expression changes, including a life-long increase in hippocampal Hdac4 expression. HDAC4 enrichment at the mTOR and Gnail promoters, accompanied by decreased activatory histone acetylation marks, support a role for HDAC4 in the downregulation of specific genes following PNFlx. Coadministration of a HDAC inhibitor prevented the effects of PNFlx on Hdac4 and $m$ TOR, and blocked the emergence of depression- and anxiety-like behavior. Further, AFlx reversed the effects of PNFlx on epigenetic modifications at the Hdac4, $m T O R$, and Gnail promoters and normalized their hippocampal expression, accompanied by a rescue of depressionand anxiety-like behaviors. Finally, viral-mediated HDAC4 overexpression in adult hippocampus was sufficient to enhance depression- but not anxiety-like behavior. Together, our findings highlight the differing molecular signatures evoked by fluoxetine based on the temporal window of treatment, and implicate dysregulated HDAC4 in the behavioral effects of PNFlx.

Although previous studies have investigated gene expression changes following AFlx (Conti et al, 2007; Miller et al, 2008), we provide the first characterization of global transcriptional changes evoked by PNFlx. A comparison of PNFlx and AFlx transcriptomes indicates primarily nonoverlapping

Figure I Postnatal fluoxetine (PNFIx) evokes persistent depression- and anxiety-like behavior and alters the hippocampal transcriptome. Shown is a schematic representation for the experimental design (a), wherein PNFIx animals were tested for juvenile play, open field test (OFT) and forced swim test (FST) in adulthood, and FST and OFT at 18 months of age. PNFIx animals showed a decline in juvenile play with reduced social grooming and pouncing (b, c) but no change in follow and chase (d). In adulthood, PNFIx animals showed depressive behavior on FST with increased immobility time (e), reduced latency to the first immobility event $(f)$, and a trend $\left({ }^{\#} p=0.07\right)$ toward an increase in immobility events $(g)$. PNFlx animals in adulthood showed increased anxiety-like behavior on the OFT with increased latency to approach the center ( $h$ ), decline in time spent (i), and path length (j) at the center of the arena. Enhanced depression- and anxiety-like behavior on the FST $(k, I)$ and OFT $(\mathrm{m})$ persisted up to 18 months in PNFIx animals. Data are the mean \pm SEM ( $n=8-10$ animals per group for all ages). ${ }^{*} p<0.05$ as compared with controls (Student's t-test). Shown is a hierarchical cluster of significantly regulated (up, red; down, green) genes $(n)$ and functional enrichment of gene ontology categories $(0)$ in the microarray analysis performed on hippocampi ( $P$ ) from 2 month-old PNFIx animals. 
regulation, with enrichment of predominantly distinct gene categories. PNFlx did not influence the hippocampal expression of $B d n f, T r k B, G d n f, F G F-2, A r c$, and $N P Y$, which are robustly regulated by AFlx (Nibuya et al, 1995; Hisaoka et al, 2001; Miller et al, 2008; Christiansen et al, 2011) and contribute mechanistically to the neurogenic and

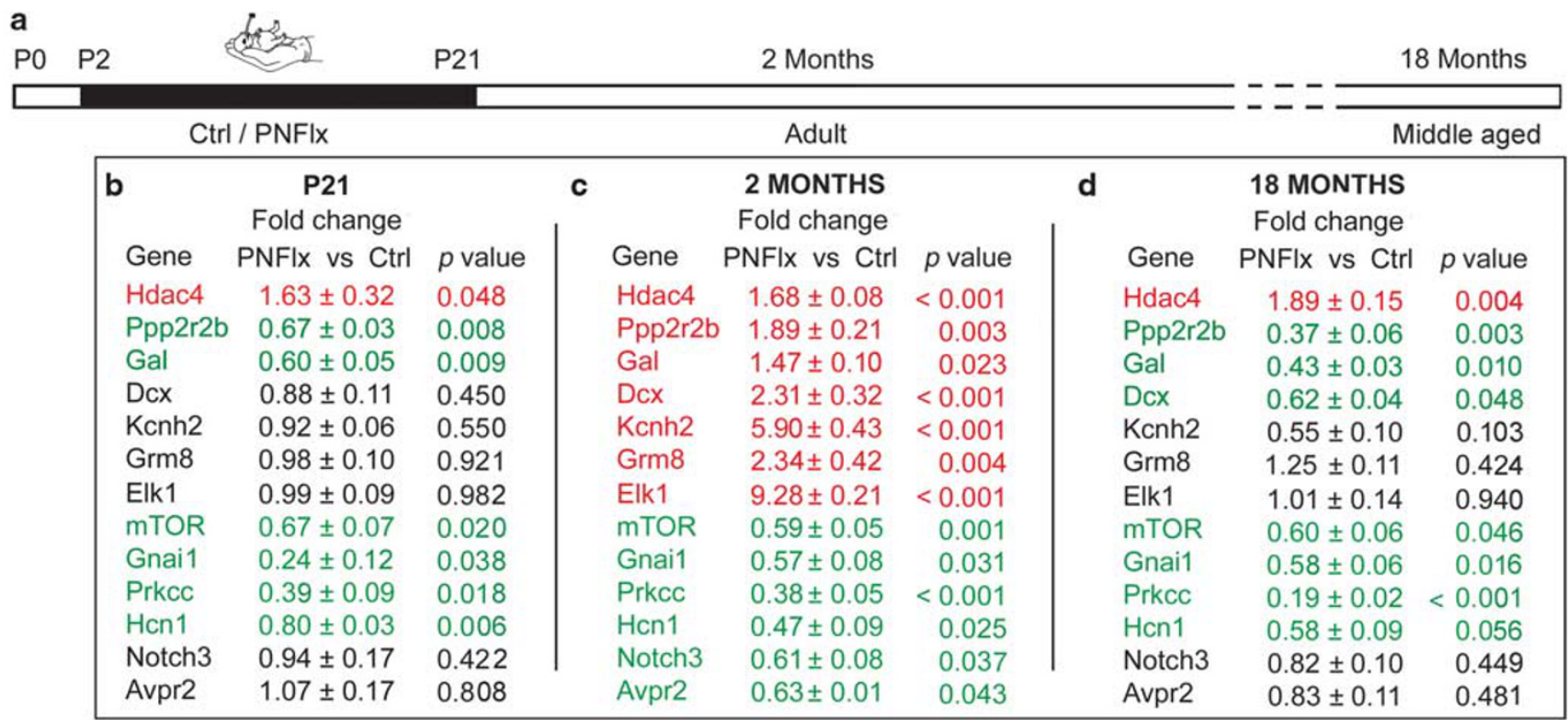
$0 . \overline{111.5}$

Ctrl PNFIx Ctrl AFIx
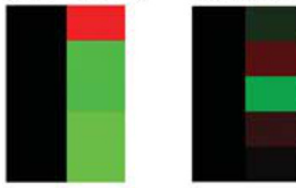

Hdac4 mTOR Gnai1 Prkcc Hen1

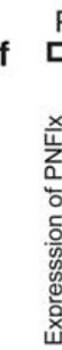

\begin{tabular}{|c|c|c|c|}
\hline$\frac{x}{4} \frac{x}{\frac{u}{L}}$ & Gene & $\begin{array}{l}\text { Fold change } \\
\text { AFIx vs Ctrl }\end{array}$ & $p$ value \\
\hline$\frac{2}{a}=\frac{5}{4}$ & Hdac4 & $0.91 \pm 0.25$ & 0.83 \\
\hline & mTOR & $1.18 \pm 0.71$ & 0.84 \\
\hline 递 & Gnai1 & $0.68 \pm 0.39$ & 0.60 \\
\hline & Prkcc & $1.12 \pm 0.19$ & 0.79 \\
\hline 这 & Hen1 & $1.02 \pm 0.52$ & 0.95 \\
\hline
\end{tabular}

P0 P2 ctrl/PNFIx P21

g

\begin{tabular}{|c|c|c|c|}
\hline$x^{\frac{x}{z}}$ & Gene & $\begin{array}{l}\text { Fold change } \\
\text { PNFIx vs Ctrl }\end{array}$ & $p$ value \\
\hline$\overline{\frac{\pi}{4}} \frac{.5}{6}$ & Bdnf & $0.56 \pm 0.14$ & 0.32 \\
\hline 응 & TrkB & $0.66 \pm 0.18$ & 0.53 \\
\hline 응 용 & Gdnf & $1.06 \pm 0.18$ & 0.93 \\
\hline$\frac{0}{\frac{0}{2}} \frac{\pi}{5}$ & Arc & $0.39 \pm 0.08$ & 0.07 \\
\hline
\end{tabular}
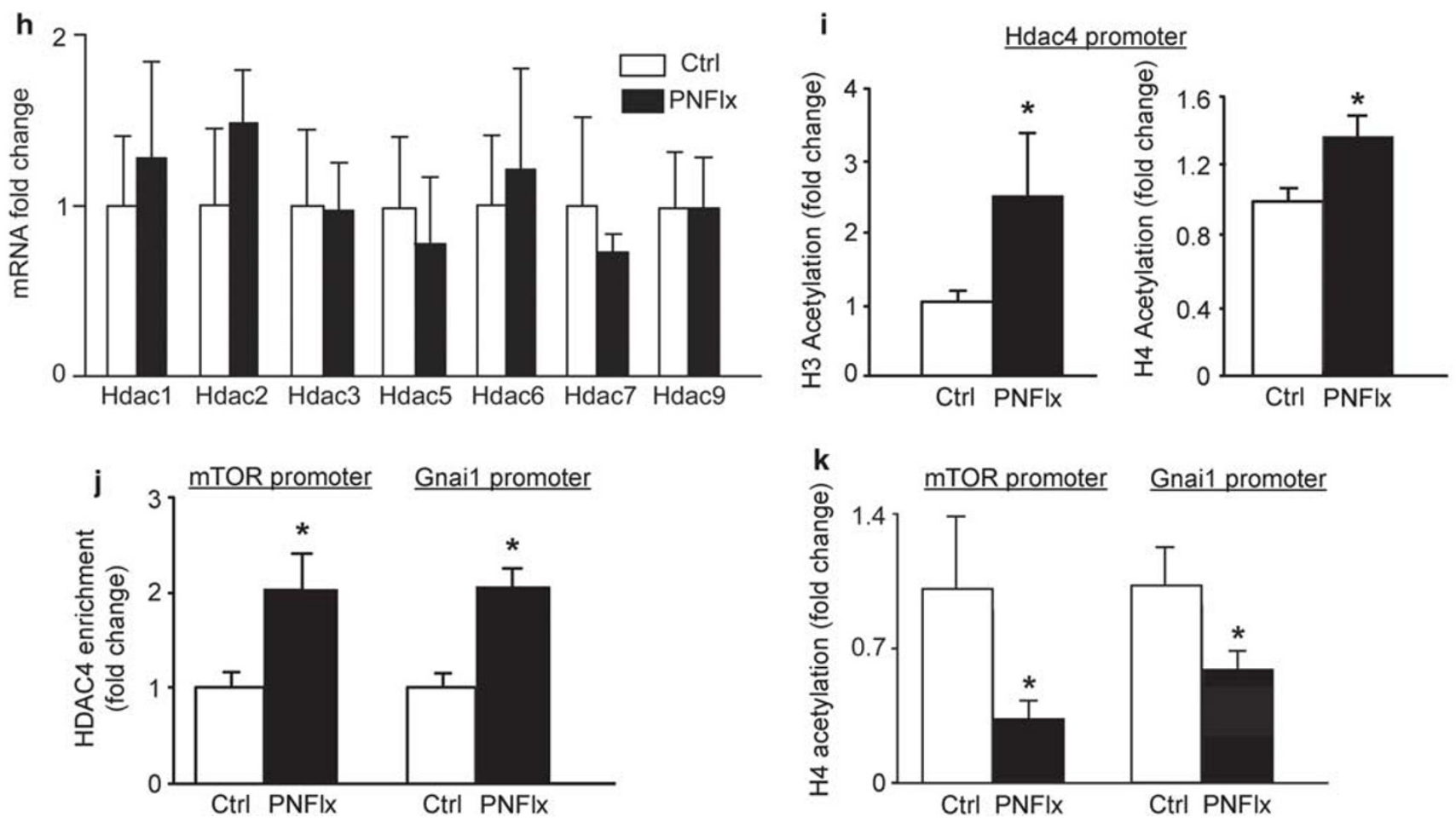
antidepressant effects of AFlx (Saarelainen et al, 2003; Sairanen et al, 2005; Christiansen et al 2011; Kohl et al, 2012). An important caveat is that the PNFlx and AFlx paradigms differ in treatment age and also the time after treatment when effects were assessed. With this in mind, we specifically examined whether select dysregulated genes observed in PNFlx animals in adulthood were altered right after the cessation of fluoxetine treatment. Our results revealed significant increases in Hdac4 expression and robust decline in mTOR, Prkcc, Hcn1, and Gnail at P21, which were maintained across life in PNFlx animals. AFlx did not influence the hippocampal expression of these genes, strengthening the evidence that fluoxetine evokes distinct molecular signatures depending on the timing of treatment. A possible reason for the differential effects of fluoxetine at these two ages could be the distinct patterns of serotonin transporter expression in the postnatal $v s$ adult brain (Narboux-Nême et al, 2008). Recent studies suggest that not only does the age of fluoxetine treatment administration influence the nature of behavioral and neuroplastic consequences (Iñiguez et al, 2010; Homberg et al, 2011), but that the time point at which these changes are assessed and duration following fluoxetine washout may also differentially affect outcomes.

The PNFlx-evoked stable increase in Hdac4 expression suggested that HDAC4-mediated transcriptional repression of specific genes may contribute to the emergence of behavioral abnormalities. Indeed, both concomitant postnatal HDAC inhibitor treatment and adult-onset fluoxetine retreatment, which prevented the emergence of depressionand anxiety-like behavior, were accompanied by normalization of Hdac4 expression. Future studies are required to address the mechanisms that mediate the PNFlx-evoked sustained increase in Hdac4 expression. Hdac4 and Hdac5 are highly expressed within the forebrain and influence plasticity (Kim et al, 2012; Sando et al, 2012) and moodrelated behavior (Hobara et al, 2010; Sailaja et al, 2012). Recent studies show anxiolytic effects in conditional, forebrain-specific HDAC4-knockout mice (Kim et al, 2012). Clinical evidence indicated elevated Hdac4 and Hdac5 mRNA in peripheral blood cells of patients with depression (Iga et al, 2007; Hobara et al, 2010). It is interesting to speculate that HDAC4, through its histonemodifying activity, may mediate the persistent downregulation of specific genes that mechanistically contribute to the behavioral phenotypes following PNFlx.

Many more genes were downregulated following PNFlx, with DNA binding and transcriptional repression being an enriched gene category. HDAC4 enrichment accompanied by reduced $\mathrm{H} 4 \mathrm{ac}$ at the $m T O R$ and Gnail promoters supports the notion that HDAC4 mediates the transcriptional repression of specific PNFlx target genes. Further, hippocampal HDAC4 overexpression also led to a decline in $m T O R$ and Gnail expression. As a target for HDAC4mediated repression in PNFlx animals, $m T O R$ is of particular interest. PNFlx animals showed decreased hippocampal $m T O R$ mRNA and protein. Normalization of PNFlx-evoked behavioral outcomes by HDAC inhibition or AFlx retreatment rescued the decreased mTOR expression. Dysregulated mTOR signaling is reported in neurogenetic (Gipson and Johnston, 2012), neurodevelopmental (Wang and Doering, 2013), and psychiatric disorders (Jernigan et al, 2011) associated with disrupted mood and social behavior. Clinical evidence indicates decreased cortical mTOR levels in depressed patients (Jernigan et al, 2011), whereas activation of mTOR signaling mediates the behavioral effects of rapid-action antidepressants ( $\mathrm{Li}$ et al, 2010). Juvenile fluoxetine treatment (P20-34), which evokes enhanced anxiety accompanied by antidepressant-like behavior, enhanced mTOR signaling in the ventral tegmental area (Warren et al, 2011). The timing of fluoxetine administration may evoke distinct and region-specific effects on the mTOR pathway. Our results suggest that decreased hippocampal mTOR, likely through an HDAC4mediated mechanism, is a stable molecular change that accompanies PNFlx-evoked depression- and anxiety-like behavior, and is normalized by treatments that rescue the behavioral effects of PNFlx.

Although HDAC4 likely contributes to the transcriptional repression of $m T O R$ and Gnail, future studies using HDAC4 ChIP-seq are required to identify the entire subset of HDAC4-regulated PNFlx target genes. Our focus on the PNFlx-mediated Hdac4 and mTOR dysregulation does not preclude a role for other factors that work in concert to generate the complex PNFlx behavioral phenotype. The reversal of molecular changes (Hdac4 and $m T O R$ ) by both postnatal SB and AFlx, distinct pharmacological strategies used in nonoverlapping temporal windows, suggests that rescue of specific molecular adaptations may underlie the blockade of PNFlx-evoked behaviors. A caveat to keep in mind is that SB is a broad spectrum HDAC inhibitor, which in addition to inhibiting HDAC4 activity would also perturb acetylation/deacetylation through other HDACs (Ajamian et al, 2004). On the basis of prior studies with valproate, which besides inhibiting HDACs evokes proteasomal

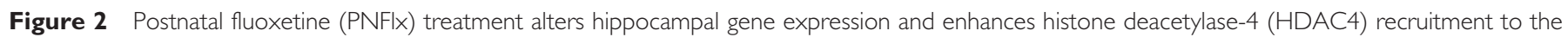

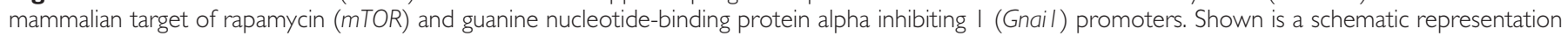

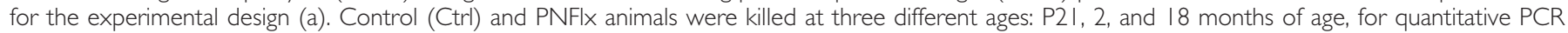

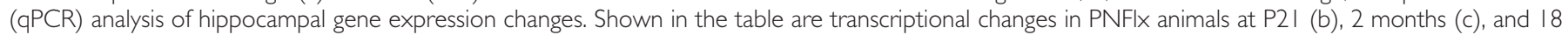

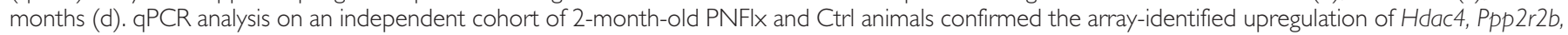

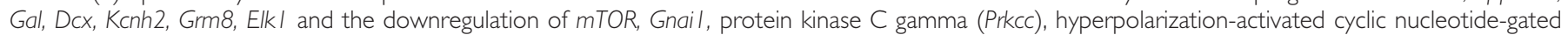

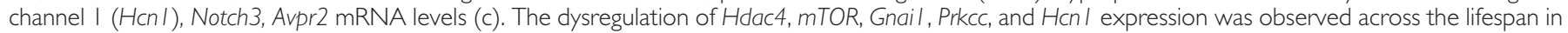

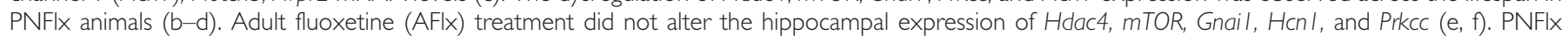

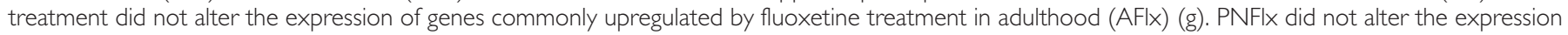

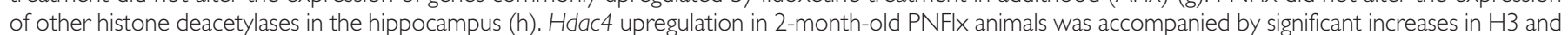

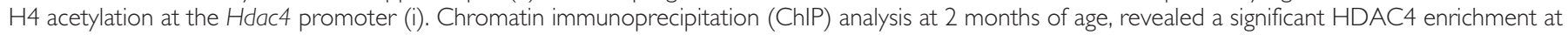

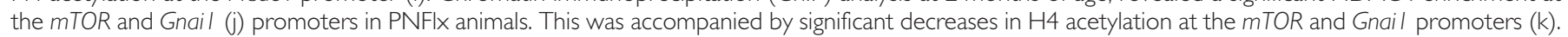
Data are expressed as fold change of control and are the mean \pm SEM ( $n=6-10$ per group). * $p<0.05$ as compared with controls (Student's $t$-test). 
a

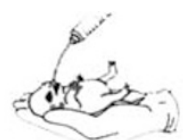

P2<smiles>[3H][Ga]I</smiles>

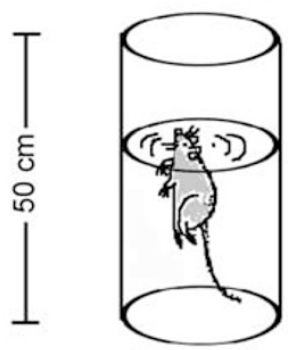

f

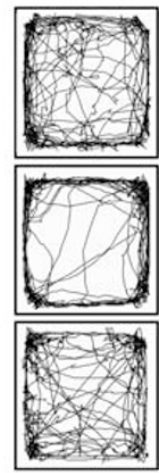

Ctrl

PNFIX

PNFIX

PNSB c

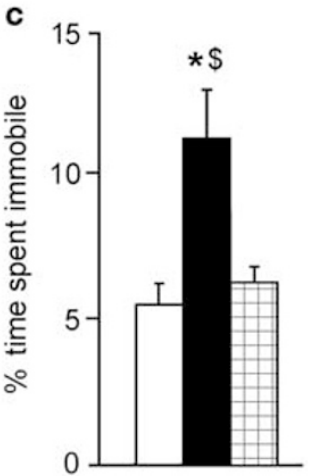

g

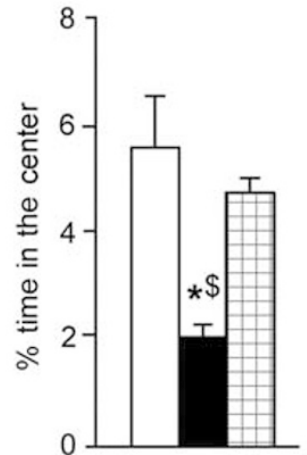

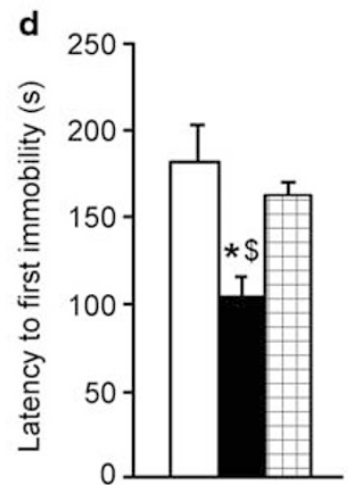

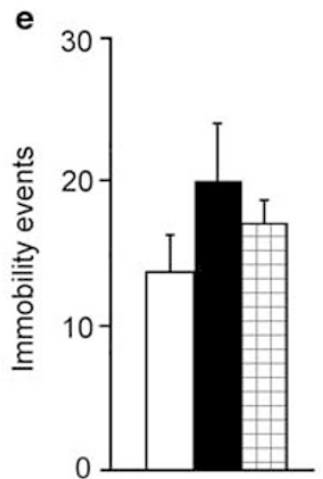

h

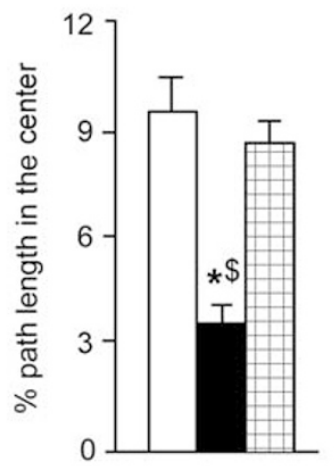

$$
\text { i }
$$

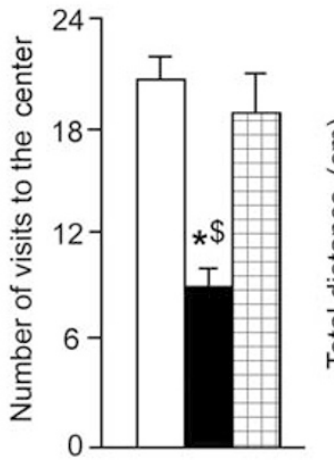

j
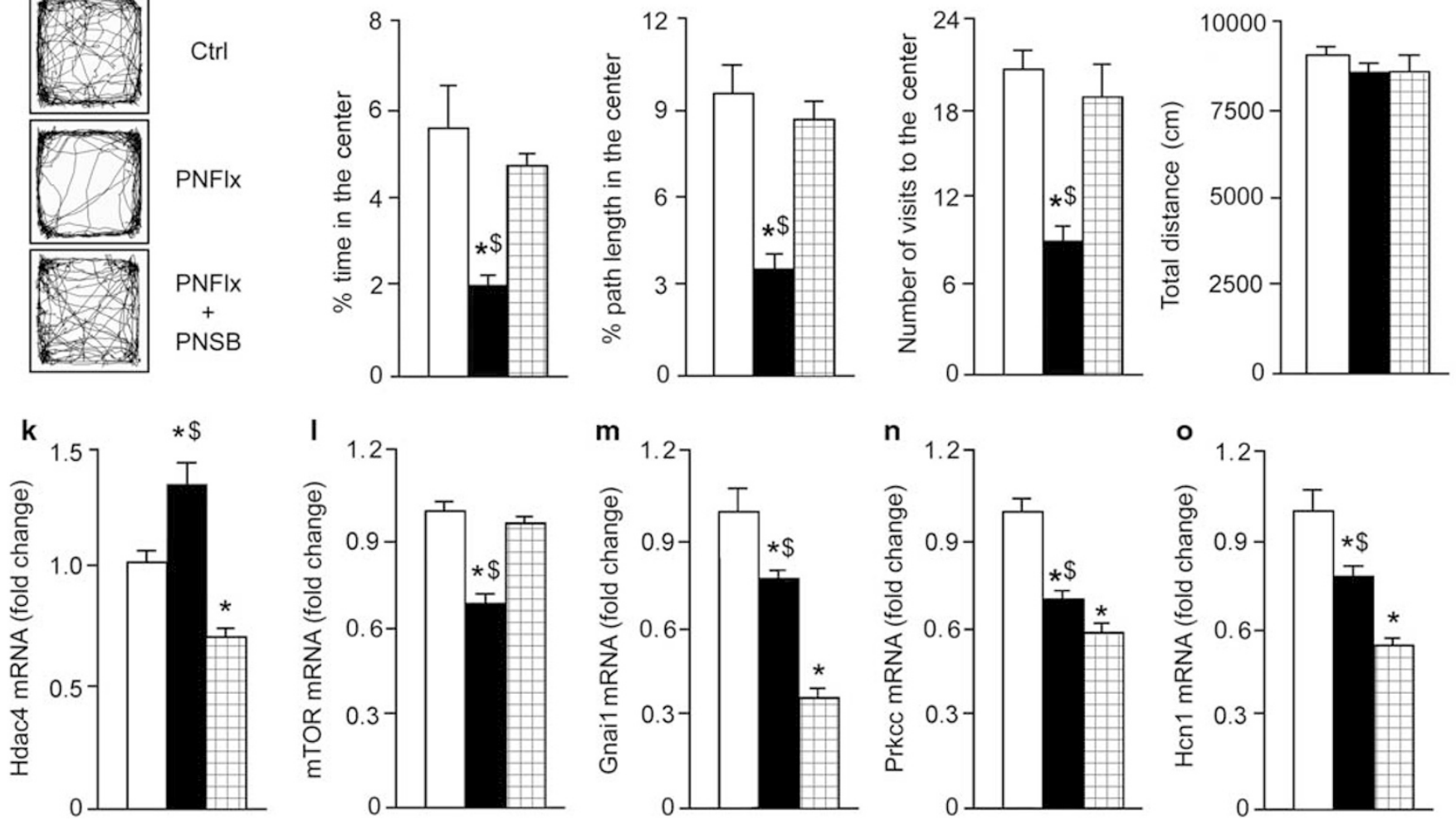

Figure 3 Postnatal treatment with sodium butyrate (SB) prevents the emergence of postnatal fluoxetine (PNFIx)-evoked molecular and behavioral changes. Shown is a schematic representation for the experimental design (a), wherein rat pups received vehicle control (Ctrl), PNFlx, or fluoxetine + sodium butyrate (PNFIx + PNSB) from P2 to P2I and were subjected to the forced swim test (FST) and open field test (OFT) in adulthood. PNFIx animals showed a significant increase in immobility time (c) and a decreased latency to first immobility (d) on the FST (b). Coadministration of the HDAC inhibitor sodium butyrate prevented the emergence of depressive behavior on the FST ( $c$ and d). Number of immobility events remained unaltered between groups (e). Shown are representative tracks in the OFT arena from Ctrl, PNFIx, and PNFIx + PNSB animals ( $f$ ). Coadministration of sodium butyrate prevented the anxiety-like behavior shown by PNFIx animals on the OFT on measures of percent time (g), path length (h), and number of visits to the center of the arena (i). Total distance moved in the arena was unaltered across groups (j). Data are the mean \pm SEM. ( $n=6-8$ per group, ${ }^{*} p<0.05$ as compared with Ctrls, $\$_{p}<0.05$ as compared with PNFIx + PNSB, one-way ANOVA, the Student-Newman-Keuls post hoc test). Coadministration of sodium butyrate also prevented the PNFlx-evoked regulation of histone deacetylase-4 (Hdac4; k) and mammalian target of rapamycin (mTOR; l), but not of guanine nucleotidebinding protein alpha inhibiting I (Gnail; m), protein kinase C gamma (Prkcc; n), and hyperpolarization-activated cyclic nucleotide-gated channel I (Hcn I; o). ( $n=6-10$ per group, ${ }^{*} p<0.05$ as compared with Ctrls, ${ }^{\$} p<0.05$ as compared with PNFIx + PNSB, one-way ANOVA, Student-Newman-Keul's post hoc test). 
a

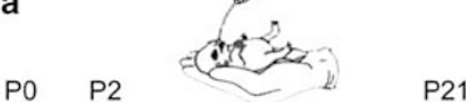

OFT, FST
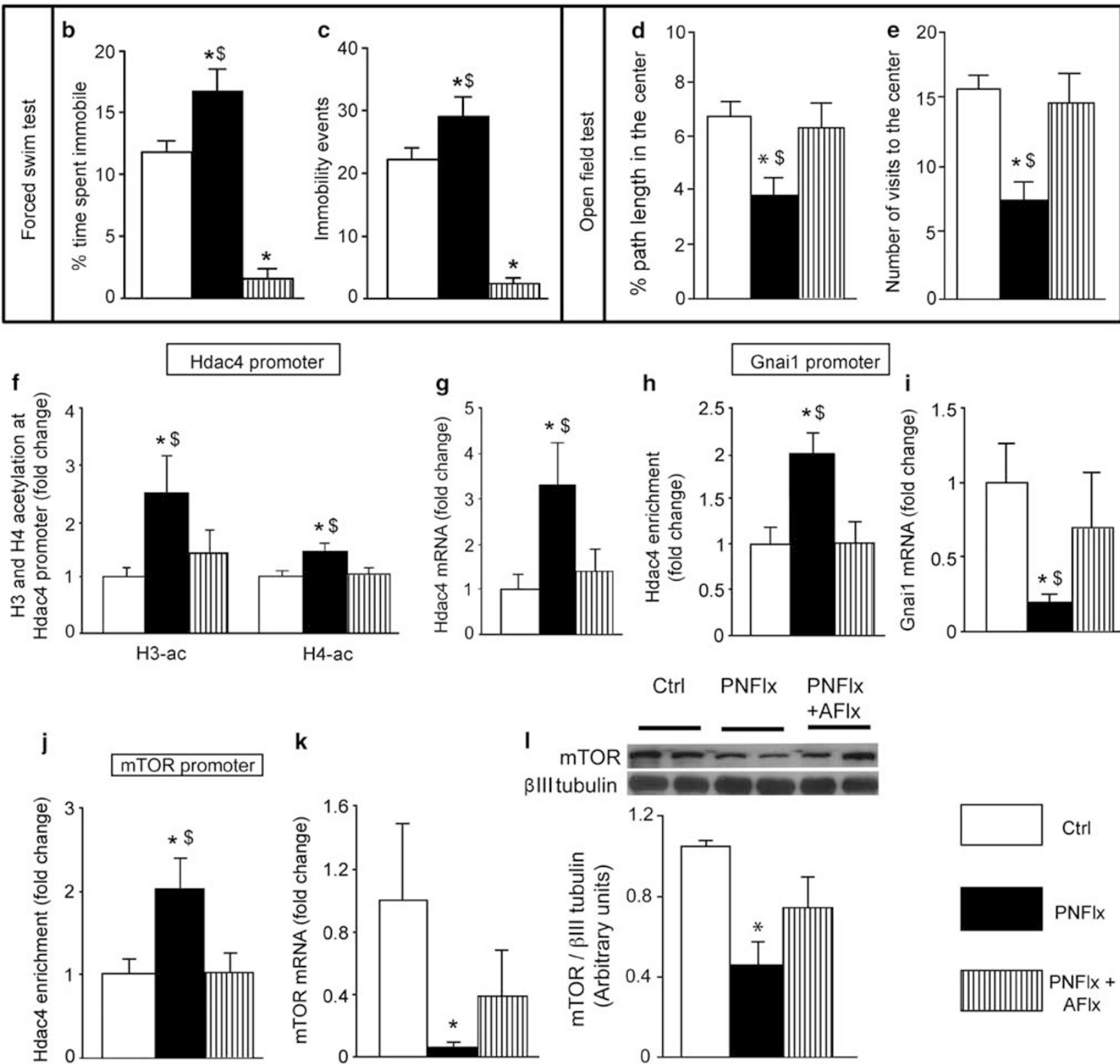

k
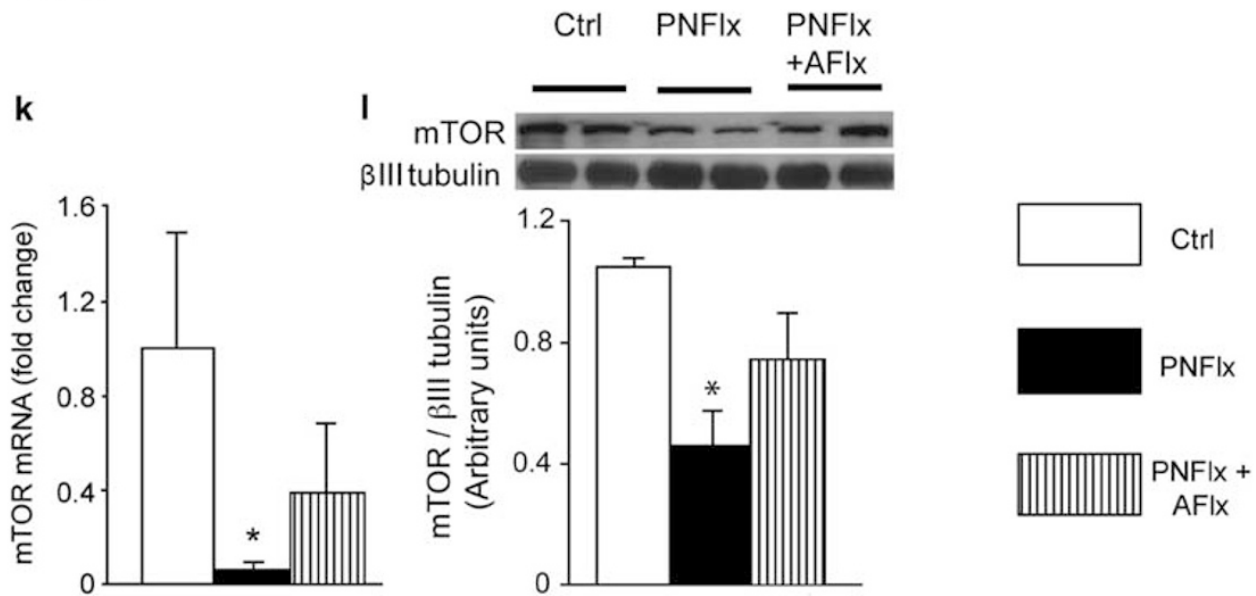

Figure 4 Adult fluoxetine (AFIx) treatment reverses behavioral and specific molecular signatures associated with postnatal fluoxetine (PNFIx). Shown is a schematic representation of the experimental design (a) wherein PNFlx animals in adulthood received vehicle or fluoxetine to give rise to three groups (control (Ctrl), PNFlx, and PNFIx + AFIx). Retreatment in adulthood with fluoxetine rescued the depressive- and anxiety-like behavior noted on the forced swim test (FST; b, c) and open field test (OFT; $d, e$ ) in PNFIx animals. PNFIx + AFlx animals did not show the PNFlx-evoked increase in immobility time (b) and number of immobility events (c), rather they exhibited antidepressant-like behavior. PNFIx + AFlx animals did not show the PNFlx-evoked anxiety on the OFT on percent path length (d) and number of visits to the center of the arena (e). Further, retreatment in adulthood with fluoxetine also ameliorated the PNFlx-evoked increase in $\mathrm{H} 3$ and $\mathrm{H} 4$ acetylation at the histone deacetylase-4 (Hdac4) promoter (f), rescued the PNFlx-mediated increase in hippocampal Hdac4 expression (g), normalized HDAC4 enrichment at the guanine nucleotide-binding protein alpha inhibiting I (Gnail; h) and mammalian target of rapamycin (mTOR; j) promoters, and attenuated the decline in Gnail (i) and mTOR (k) expression. Western blot analysis indicated that the PNFlxevoked decline in mTOR levels was attenuated in the PNFIx + AFIx group (I). Data are the mean \pm SEM. $(n=6-8$ per group for FST; $n=9-10$ per group for OFT; $n=6-10$ for ChIP/quantitative PCR (qPCR) analysis, and $n=4$ for western blot analysis. ${ }^{*} p<0.05$ compared with controls, ${ }^{\$} p<0.05$ compared with PNFIx + AFlx, one-way ANOVA, Student-Newman-Keul's post hoc test). 


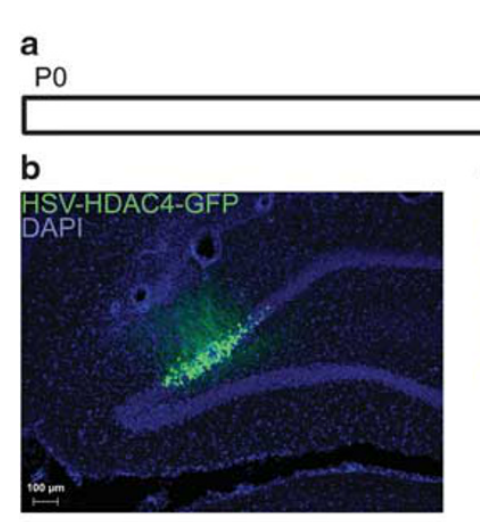

d

C
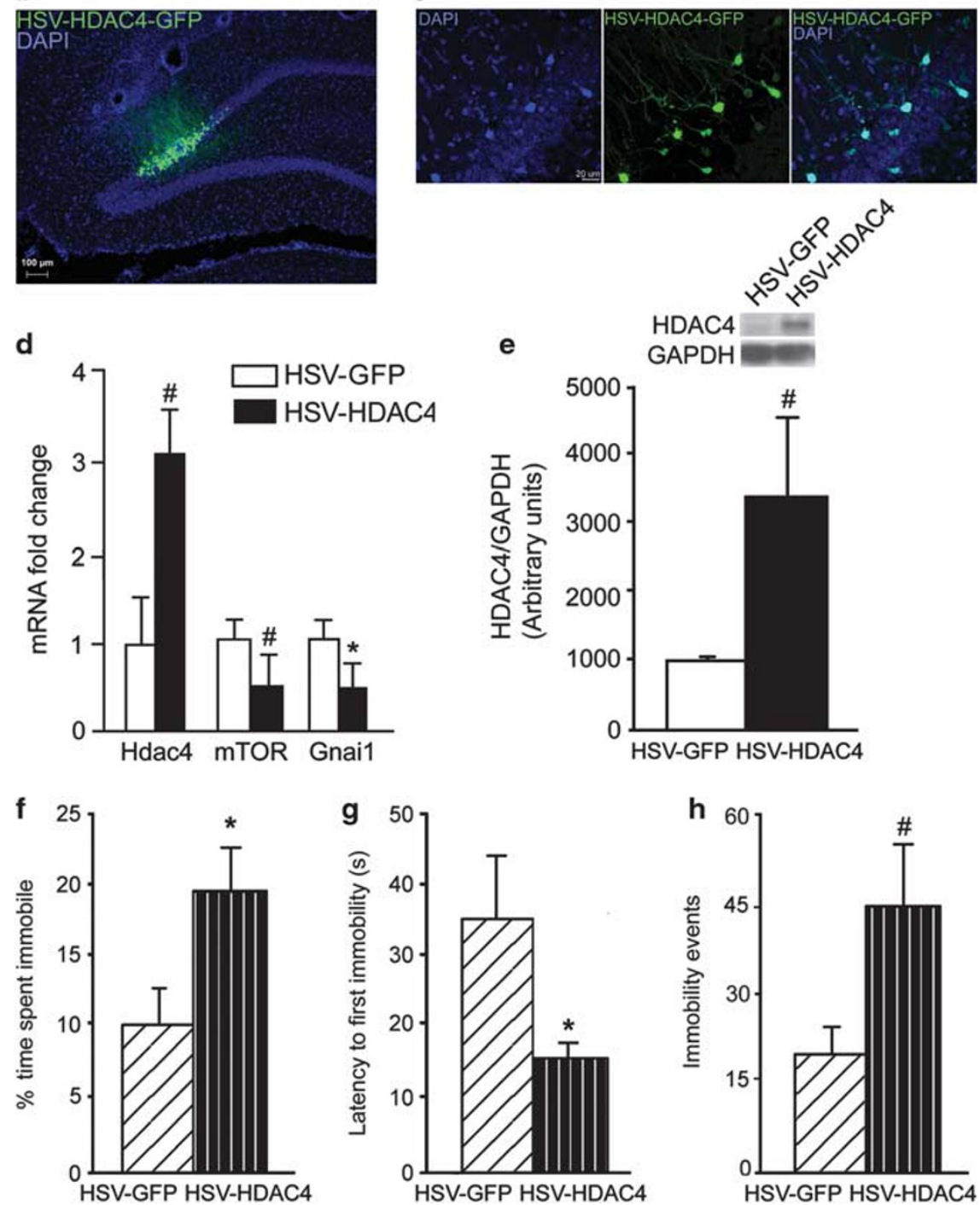

Figure 5 Hippocampal histone deacetylase-4 (Hdac4) overexpression in adulthood evokes depression-like behavior. Shown is the schematic for the experimental paradigm (a) wherein adult rats received bilateral stereotactic injections into the hippocampus of bicistronic construct HSV-HDAC4-GFP or HSV-GFP and were subjected to the forced swim test (FST). Shown are representative images for HSV-HDAC4-GFP (green) and DAPI (blue) expression in the hippocampus (b) and GFP-DAPI colocalization in the dentate gyrus (c). Quantitative PCR (qPCR) and western analysis confirmed HDAC4 overexpression with a trend noted toward significance in the qPCR $(p=0.07 ; d)$ and western blot results $(p=0.062 ;$ e). HDAC4 overexpression resulted in a significant decline in guanine nucleotide-binding protein alpha inhibiting I (Gnail) expression and a strong trend $(p=0.05 \mathrm{I}$ ) for a decline in mammalian target of rapamycin (mTOR) expression. HDAC4 overexpression significantly increased percent immobility time ( $f$ ) and reduced latency to the first immobility event $(g)$ in the FST. A strong trend $(p=0.07)$ toward increase in the number of immobility events was noted in HDAC4-GFP-overexpressing animals (e). Data are the mean \pm SEM ( $n=6-7$ animals per group for behavioral studies; $n=4$ for qPCR and western blot analysis). $* p<0.05, \# p<0.10$ as compared with HSV-GFP (Student's t-test).

degradation of HDAC2 (Kramer et al, 2003), we speculate that a decline in overall HDAC4 levels in PNFlx + PNSB animals could contribute to the normalization of mTOR expression. Our results indicate that co-treatment with SB prevents the emergence of PNFlx-evoked depression- and anxiety-like behavior. Prior reports of SB treatment to adult animals indicate an augmentation of the behavioral effects of SSRIs (Schroeder et al, 2007). This suggests contrasting effects of HDAC inhibition on the behavioral effects of fluoxetine based on the temporal window of treatment.

Our findings with AFlx retreatment of PNFlx animals indicate that interventions in adulthood can also counteract negative behavioral outcomes following PNFlx. These results corroborate prior evidence that $\mathrm{AFlx}$ rescues depression- and anxiety-like behavior in PNFlx animals (Karpova et al, 2009). AFlx-mediated rescue of behavior was accompanied by normalization of Hdac4, mTOR, and Gnail 
expression, and a reversal of HDAC4 enrichment at the $m T O R$ and Gnail promoters. AFlx itself did not influence Hdac4, $m T O R$, and Gnail expression, raising the intriguing possibility that AFlx influences Hdac4 expression and HDAC4-regulated targets only in the context of prior PNFlx exposure. A previous study indicates that the antidepressant imipramine downregulates HDAC5 in socially defeated animals but not in controls (Tsankova et al, 2006), further indicating that antidepressant-evoked molecular changes are context dependent. Importantly, evidence that AFlx can rescue PNFlx-induced behavioral abnormalities suggests that such changes, although potentially life long, are not immutable and can be influenced in adulthood by therapeutic strategies.

Our studies with viral-mediated hippocampal HDAC4 overexpression showed a significant increase in depressionlike behavior in the FST. Given the lasting increases across the life span noted in hippocampal Hdac4 expression following PNFlx, our results suggest that such enhanced HDAC4 may mediate effects on depression-like symptoms noted in animals with a history of PNFlx. Although we did not observe increased anxiety-like behavior following HDAC4 overexpression, our results do not allow us to rule out a role for HDAC4 in PNFlx-evoked anxiety, as earlyonset changes in HDAC4 may be required for the anxiogenic effects of PNFlx. HDAC4 overexpression in adulthood in treatment-naive animals resulted in a decline in hippocampal $m T O R$ and Gnai1 levels, further lending credence to the hypothesis that HDAC4 mediates the regulation of specific PNFlx target genes.

In summary, we provide evidence of molecular signatures selectively associated with fluoxetine treatment in postnatal life, a temporal window wherein treatment evokes persistent depression- and anxiety-like behaviors. We posit that HDAC4 induction through effects on chromatin remodeling results in the establishment and maintenance of stable molecular abnormalities that contribute to the behavioral phenotypes of PNFlx. Further, we demonstrate that specific molecular changes, as well as depression- and anxiety-like behaviors, are rescued by potential therapeutic interventions in postnatal or adult life. Our results provide novel leads for the development of new approaches for treating a range of depression and anxiety syndromes, and support the building evidence that HDAC4 is a putative drug target for antidepressant therapy.

\section{FUNDING AND DISCLOSURE}

This research was supported by a TIFR intramural grant (VAV), support from the Department of Biotechnology, Center of Excellence in Epigenetics, IISER (VAV; BT/01/ COE/09/07), and a grant from the National Institute of Mental Health (EJN; P50MH096890). The authors declare no conflict of interest.

\section{ACKNOWLEDGEMENTS}

We acknowledge Brigette Pinheiro and Sapna Narayan for technical assistance and Professor John McGrath and Dr Thomas Burne, the University of Queensland for inputs on statistical analysis.

\section{REFERENCES}

Ajamian F, Salminen A, Reeben M (2004). Selective regulation of class I and class II histone deacetylases expression by inhibitors of histone deacetylases in cultured mouse neural cells. Neurosci Lett 365: 64-68.

Ansorge MS, Zhou M, Lira A, Hen R, Gingrich JA (2004). Early-life blockade of the 5-HT transporter alters emotional behavior in adult mice. Science 306: 879-881.

Ansorge MS, Morelli E, Gingrich JA (2008). Inhibition of serotonin but not norepinephrine transport during development produces delayed, persistent perturbations of emotional behaviors in mice. J Neurosci 28: 199-207.

Benekareddy M, Goodfellow NM, Lambe EK, Vaidya VA (2010). Enhanced function of prefrontal serotonin 5-HT(2) receptors in a rat model of psychiatric vulnerability. J Neurosci 30: 12138-12150.

Benjamini Y, Hochberg Y (1995). Controlling the false discovery rate: a practical and powerful approach to multiple testing. $J$ R Statist Soc B 57: 289-300.

Bookout AL, Mangelsdorf DJ (2003). Quantitative real-time PCR protocol for analysis of nuclear receptor signaling pathways. Nucl Recept Signal 1: e102.

Brambilla P, Cipriani A, Hotopf M, Barbui C (2005). Side-effect profile of fluoxetine in comparison with other SSRIs, tricyclic and newer antidepressants: a meta-analysis of clinical trial data. Pharmacopsychiatry 38: 69-77.

Christiansen SH, Olesen MV, Wortwein G, Woldbye DP (2011). Fluoxetine reverts chronic restraint stress-induced depressionlike behaviour and increases neuropeptide $\mathrm{Y}$ and galanin expression in mice. Behav Brain Res 216: 585-591.

Clark MS, Sexton TJ, McClain M, Root D, Kohen R, Neumaier JF (2002). Overexpression of 5-HT1B receptor in dorsal raphe nucleus using Herpes Simplex virus gene transfer increases anxiety behavior after inescapable stress. J Neurosci 22: 4550-4562.

Conti B, Maier R, Barr AM, Morale MC, Lu X, Sanna PP et al (2007). Region-specific transcriptional changes following the three antidepressant treatments electro convulsive therapy, sleep deprivation and fluoxetine. Mol Psychiatry 12: 167-189.

Deacon RM, Bannerman DM, Rawlins JN (2002). Anxiolytic effects of cytotoxic hippocampal lesions in rats. Behav Neurosci 116: 494-497.

Dennis G, Sherman BT, Hosack DA, Yang J, Gao W, Lane HC et al (2003). DAVID: Database for Annotation, Visualization, and Integrated Discovery. Genome Biol 4: 3.

Dulawa SC, Holick KA, Gundersen B, Hen R (2004). Effects of chronic fluoxetine in animal models of anxiety and depression. Neuropsychopharmacology 29: 1321-1330.

Gipson TT, Johnston MV (2012). Plasticity and mTOR: towards restoration of impaired synaptic plasticity in mTOR-related neurogenetic disorders. Neural Plast 2012: 486402.

Hisaoka K, Nishida A, Koda T, Miyata M, Zensho H, Morinobu S et al (2001). Antidepressant drug treatments induce glial cell line-derived neurotrophic factor (GDNF) synthesis and release in rat C6 glioblastoma cells. J Neurochem 79: 25-34.

Hobara T, Uchida S, Otsuki K, Matsubara T, Funato H, Matsuo K et al (2010). Altered gene expression of histone deacetylases in mood disorder patients. J Psychiatr Res 44: 263-270.

Holmes A, Murphy DL, Crawley JN (2003). Abnormal behavioral phenotypes of serotonin transporter knockout mice: parallels with human anxiety and depression. Biol Psychiatry 54: 953-959.

Homberg JR, Schiepers OJ, Schoffelmeer AN, Cuppen E, Vanderschuren LJ (2007). Acute and constitutive increases in central serotonin levels reduce social play behaviour in peri-adolescent rats. Psychopharmacology (Berl) 195: 175-182.

Homberg JR, Olivier JD, Blom T, Arentsen T, van Brunschot C, Schipper P et al (2011). Fluoxetine exerts age-dependent effects on behavior and amygdala neuroplasticity in the rat. PLoS One 6: e16646. 
Iga J, Ueno S, Yamauchi K, Numata S, Kinouchi S, TayoshiShibuya $S$ et al (2007). Altered HDAC5 and CREB mRNA expressions in the peripheral leukocytes of major depression. Prog Neuropsychopharmacol Biol Psychiatry 31: 628-632.

Iñiguez SD, Warren BL, Bolaños-Guzmán CA (2010). Short- and long-term functional consequences of fluoxetine exposure during adolescence in male rats. Biol Psychiatry 67: 1057-1066.

Jernigan CS, Goswami DB, Austin MC, Iyo AH, Chandran A, Stockmeier CA et al (2011). The mTOR signaling pathway in the prefrontal cortex is compromised in major depressive disorder. Prog Neuropsychopharmacol Biol Psychiatry 35: 1774-1779.

Karpova NN, Lindholm J, Pruunsild P, Timmusk T, Castren E (2009). Long-lasting behavioural and molecular alterations induced by early postnatal fluoxetine exposure are restored by chronic fluoxetine treatment in adult mice. Eur Neuropsychopharmacol 19: 97-108.

Kheirbek MA, Drew LJ, Burghardt NS, Costantini DO, Tannenholz L, Ahmari SE et al (2013). Differential control of learning and anxiety along the dorsoventral axis of the dentate gyrus. Neuron 77: 955-968.

Kim HJ, Leeds P, Chuang DM (2009). The HDAC inhibitor, sodium butyrate, stimulates neurogenesis in the ischemic brain. I Neurochem 110: 1226-1240.

Kim MS, Akhtar MW, Adachi M, Mahgoub M, Bassel-Duby R, Kavalali ET et al (2012). An essential role for histone deacetylase 4 in synaptic plasticity and memory formation. J Neurosci 32: 10879-10886.

Kiryanova V, Smith VM, Dyck RH, Antle MC (2013). The effects of perinatal fluoxetine treatment on the circadian system of the adult mouse. Psychopharmacology 225: 743-751.

Kodish I, Rockhill C, Varley C (2011). Pharmacotherapy for anxiety disorders in children and adolescents. Dialogues Clin Neurosci 13: 439-452.

Kohl Z, Winner B, Ubhi K, Rockenstein E, Mante M, Münch M et al (2012). Fluoxetine rescues impaired hippocampal neurogenesis in a transgenic A53T synuclein mouse model. Eur J Neurosci 35: 10-19.

Kramer OH, Zhu P, Ostendorff HP, Golebiewski M, Tiefenbach J, Peters MA et al (2003). The histone deacetylase inhibitor valproic acid selectively induces proteasomal degradation of HDAC2. EMBO J 22: 3411-3420.

Li N, Lee B, Liu RJ, Banasr M, Dwyer JM, Iwata M et al (2010). mTOR-dependent synapse formation underlies the rapid antidepressant effects of NMDA antagonists. Science 329: 959-964.

Lira A, Zhou M, Castanon N, Ansorge MS, Gordon JA, Francis JH et al (2003). Altered depression-related behaviors and functional changes in the dorsal raphe nucleus of serotonin transporterdeficient mice. Biol Psychiatry 54: 960-971.

Maciag D, Simpson KL, Coppinger D, Lu Y, Wang Y, Lin RC et al (2006). Neonatal antidepressant exposure has lasting effects on behavior and serotonin circuitry. Neuropsychopharmacology 31: 47-57.

Miller BH, Schultz LE, Gulati A, Cameron MD, Pletcher MT (2008). Genetic regulation of behavioral and neuronal responses to fluoxetine. Neuropsychopharmacology 33: 1312-1322.

Narboux-Nême N, Pavone LM, Avallone L, Zhuang X, Gaspar P (2008). Serotonin transporter transgenic (SERTcre) mouse line reveals developmental targets of serotonin specific reuptake inhibitors (SSRIs). Neuropharmacology 55: 994-1005.

Nibuya M, Morinobu S, Duman RS (1995). Regulation of BDNF and trkB mRNA in rat brain by chronic electroconvulsive seizure and antidepressant drug treatments. J Neurosci 15: 7539-7547.

Norcross M, Mathur P, Enoch AJ, Karlsson RM, Brigman JL, Cameron HA et al (2008). Effects of adolescent fluoxetine treatment on fear-, anxiety- or stress-related behaviors in C57BL/6J or BALB/CJ mice. Psychopharmacology 200: 413-424.

Olivier JD, Blom T, Arentsen T, Homberg JR (2011). The agedependent effects of selective serotonin reuptake inhibitors in humans and rodents: a review. Prog Neuropsychopharmacol Biol Psychiatry 35: 1400-1408.

Popa D, Lena C, Alexandre C, Adrien J (2008). Lasting syndrome of depression produced by reduction in serotonin uptake during postnatal development: evidence from sleep, stress, and behavior. J Neurosci 28: 3546-3554.

Renthal W, Maze I, Krishnan V, Covington HE 3rd, Xiao G, Kumar A et al (2007). Histone deacetylase 5 epigenetically controls behavioral adaptations to chronic emotional stimuli. Neuron 56: 517-529.

Saarelainen T, Hendolin P, Lucas G, Koponen E, Sairanen M, MacDonald E et al (2003). Activation of the TrkB neurotrophin receptor is induced by antidepressant drugs and is required for antidepressant-induced behavioral effects. J Neurosci 23: 349-357.

Sailaja BS, Cohen-Carmon D, Zimmerman G, Soreq H, Meshorer E (2012). Stress-induced epigenetic transcriptional memory of acetylcholinesterase by HDAC4. Proc Natl Acad Sci USA 109: E3687-E3695.

Sairanen M, Lucas G, Ernfors P, Castren M, Castren E (2005). Brain-derived neurotrophic factor and antidepressant drugs have different but coordinated effects on neuronal turnover, proliferation, and survival in the adult dentate gyrus. J Neurosci 25: 1089-1094.

Sando R, Gounko N, Pieraut S, Liao L, Yates J 3rd, Maximov A (2012). HDAC4 governs a transcriptional program essential for synaptic plasticity and memory. Cell 151: 821-834.

Schroeder FA, Lin CL, Crusio WE, Akbarian S (2007). Antidepressant-like effects of the histone deacetylase inhibitor, sodium butyrate, in the mouse. Biol Psychiatry 62: 55-64.

Silva CM, Goncalves L, Manhaes-de-Castro R, Nogueira MI (2010). Postnatal fluoxetine treatment affects the development of serotonergic neurons in rats. Neurosci Lett 483: 179-183.

Tsankova NM, Berton O, Renthal W, Kumar A, Neve RL, Nestler EJ (2006). Sustained hippocampal chromatin regulation in a mouse model of depression and antidepressant action. Nat Neurosci 9: 519-525.

Ward RK, Zamorski MA (2002). Benefits and risks of psychiatric medications during pregnancy. Am Fam Physician 66: 629-636.

Warren BL, Iñiguez SD, Alcantara LF, Wright KN, Parise EM, Weakley SK et al (2011). Juvenile administration of concomitant methylphenidate and fluoxetine alters behavioral reactivity to reward- and mood-related stimuli and disrupts ventral tegmental area gene expression in adulthood. J Neurosci 31: 10347-10358.

Wang H, Doering LC (2013). Reversing autism by targeting downstream mTOR signaling. Front Cell Neurosci 7: 28.

Supplementary Information accompanies the paper on the Neuropsychopharmacology website (http://www.nature.com/npp) 